\title{
Control over phase separation and nucleation using a optical-tweezing potential
}

\author{
Klaas Wynne, Finlay Walton
}

Klaas Wynne, Finlay Walton, "Control over phase separation and nucleation using a optical-tweezing potential," Proc. SPIE 10723, Optical Trapping and Optical Micromanipulation XV, 1072300 (7 September 2018); doi: 10.1117/12.2324119

Event: SPIE Nanoscience + Engineering, 2018, San Diego, California, United States 


\title{
Control over phase separation and nucleation using an optical- tweezing potential
}

\author{
Klaas Wynne* and Finlay Walton \\ School of Chemistry, University of Glasgow, UK
}

\begin{abstract}
Control over the nucleation of new phases is highly desirable but elusive. Even though there is a long history of crystallization engineering by varying physicochemical parameters, controlling which polymorph crystallizes or whether a molecule crystallizes or forms an amorphous precipitate is still a black art. Although there are now numerous examples of control using laser-induced nucleation, a physical understanding is absent and preventing progress. We will show that concentration fluctuations in the neighborhood of a liquid-liquid critical point can be harnessed by an optical-tweezing potential to induce concentration gradients. A simple theoretical model shows that the stored electromagnetic energy of the laser beam produces a free-energy potential that forces phase separation or triggers the nucleation of a new phase. Experiments in liquid mixtures using a low-power laser diode confirm the effect. Phase separation and nucleation through an optical-tweezing potential explains the physics behind non-photochemical laser-induced nucleation and suggests new ways of manipulating matter.
\end{abstract}

Keywords: nucleation, critical fluctuations, optical tweezers, phase separation, control, crystallization, liquids, critical point

\section{INTRODUCTION}

In the late 1990s and early 2000s, it was shown that a nanosecond laser can be used to induce nucleation of crystals in a supersaturated solution through a non-photochemical process. ${ }^{1}$ Most excitingly, it was reported that the laser polarization could be used to control which polymorph would nucleate, promising an unprecedented degree of control. ${ }^{2,3}$ Subsequent work showed that laser pulses can induce nucleation of various crystals, ${ }^{4-8}$ liquid crystals, ${ }^{9,10}$ and bubbles. ${ }^{11}$ However, these nanosecond-laser nucleation experiments defied all explanation. ${ }^{12-14}$

A series of publications has shown that optical tweezing can be used to nucleate crystals from super-saturated ${ }^{15-18}$ and strangely even from under-saturated solutions. ${ }^{19}$ Optical tweezing is a technique used widely in physics and biology and involves the trapping of a high-refractive-index particle through optical forces. ${ }^{20}$ Thus, it was assumed that in these experiments the laser was tweezing pre-existing clusters or pre-nuclei. ${ }^{16}$ However, these optical tweezing nucleation experiments were only shown to work on a liquid-gas interface. This strongly suggest that heating, evaporation, convection, and Marangoni effects play critical roles, ${ }^{21}$ which would also explain why the method works in undersaturated solutions. These experiments also demonstrated polymorph selection. ${ }^{15,19}$ As in the case of non-photochemical laser-induced nucleation, no sensible theory is available to describe the physics of these results. Thus, it is fair to say that a physical understanding of all of these phenomena is still sorely lacking.

A possible explanation for these laser-induced nucleation experiments might come from thermodynamics. According to classical Gibbs nucleation theory, nucleation is impeded by the fact that a growing nucleus has an energeticallyunfavorable interface that results in a barrier on the way to the crystalline state. ${ }^{22,23}$ This means that in a supersaturated solution or supercooled liquid, the crystal is the thermodynamically most stable state but - in the absence of heterogeneous nucleation sites - can only be accessed through random fluctuations leading to a nucleus exceeding a critical size. A number of recent experiments have called into question the validity of classical Gibbs nucleation theory and invoked the presence of so-called pre-nucleation clusters. ${ }^{24-26}$ Although there have been experimental studies reporting pre-nucleation clusters, ${ }^{27,28}$ they are still considered controversial. ${ }^{29,30}$

*klaas.wynne@glasgow.ac.uk; www.wijnne.com

Optical Trapping and Optical Micromanipulation XV, edited by Kishan Dholakia, Gabriel C. Spalding, Proc. of SPIE Vol. 10723, $1072300 \cdot$ C 2018 SPIE · CCC code: 0277-786X/18/\$18 · doi: 10.1117/12.2324119 
However, a paper by Frenkel on protein crystallization showed that nucleation is sped up, not simply by increasing the concentration, but by choosing a concentration that is near a (hidden) liquid-liquid demixing critical point. ${ }^{31-33}$ This is because critical concentration fluctuations give rise to liquidlike protein droplets that increase the probability of the formation of a critical nucleus. The metastable critical point idea found some traction in the chemical engineering community where it was related to the phenomenon of "oiling out" without too much detailed theoretical analysis. ${ }^{23,34,35}$ Could liquid-liquid phase separation, oiling out, pre-nucleation clusters, and laser-induced nucleation all be aspects of the same phenomenon? ${ }^{26,36,37}$

On approaching a liquid-liquid demixing critical point, concentration fluctuations increase. As a result, it should be easier for an external "force", such as optical tweezing, to manipulate the concentration locally. ${ }^{38-40}$ Optical tweezing normally involves the trapping of a high-refractive-index particle by the small forces exerted by a focused laser beam. However, a alternative view of nucleation is like a reaction where the supersaturated solution is the reactant state and the crystal (nucleus) the product state. Switching on an optical-tweezing laser will lower the free energy of the product state (even if the crystal nucleus does not exist yet), will therefore increase the driving force and lower the barrier for the nucleation process, and increase the "reaction rate". This simple but novel idea was first tested by us on liquid mixtures ${ }^{41}$ and is discussed further here.
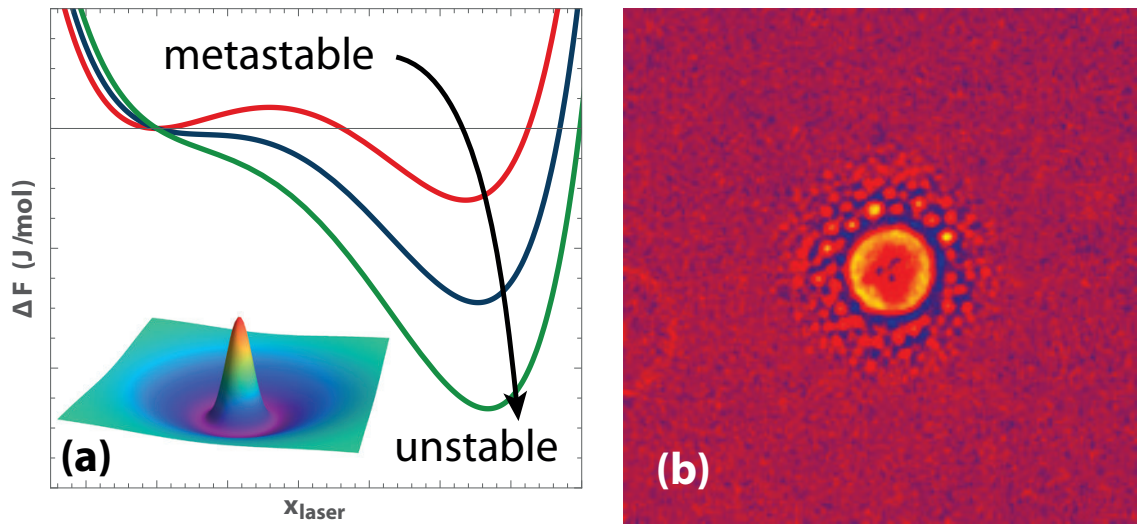

Figure 1. Principle of laser-induced phase separation (LIPS). (a) The presence of a laser in a liquid mixture near a liquidliquid critical point creates an optical trap for the high refractive-index liquid. ${ }^{41}$ (inset) This creates a high-index-liquidenriched region in the beam, surrounded by a depleted region. (b) When LIPS takes place under the right conditions, it causes nucleation of a new phase. ${ }^{41}$

A simple model of a liquid mixture based on the regular solution model ${ }^{42}$ including an extra term representing the stored electromagnetic energy of a tweezing laser, shows that the concentration-dependent free energy is indeed more easily perturbed near a critical point (Figure 1). ${ }^{41}$ Experiments were carried out on nitrobenzene-decane mixtures using a simple diode laser that showed that the optical tweezer can pull (high refractive index) nitrobenzene out of the mixture in a process named laser-induced phase separation (LIPS). ${ }^{41}$ Near the liquid-liquid binodal the laser can trigger the formation of phase separated droplets through LIPS and nucleation.

Thus, LIPS effectively harnesses critical concentration fluctuations to drive the system towards the phase separated state. It can explain all the laser-induced nucleation experiments even in the absence of an obvious critical point (that is, it may be hidden in a metastable region such as below the liquidus).

\section{RESULTS AND DISCUSSION}

The phase transition considered here occurs when two liquids are miscible at high temperature but separate into two phases when the temperature is lowered (that is, it has an upper consolute point). The free energy of such a system can be described by the regular solution model, ${ }^{42}$ which is a simple but surprisingly comprehensive physical description of the mixing and demixing of two liquids. ${ }^{41}$ The red curves in Figure 2 show the change in free energy when the mole fraction is changed in a small sub-volume in the sample. When a laser is focused into the small sub-volume, an additional energy term $-n^{2} I$ (where $n$ is the refractive index and $I$ the laser intensity) must be added representing the total stored electromagnetic energy, which gives rise to an optical-tweezing potential ${ }^{20}$ that will tend to pull in the liquid with the highest refractive index. 
The blue and green curves in Figure 2 show the predicted effect of the additional optical-tweezing term. In the mixed regime, this will give rise to laser-induced phase separation (LIPS), while in the metastable regime, the laser can trigger nucleation. Our calculations predict that these effects would occur at very reasonable laser powers of $10-100 \mathrm{~mW} .{ }^{41}$ Simulations show that the high refractive-index liquid is drawn into the laser volume at a rate limited by diffusion leaving behind a depleted volume that slowly fills up by diffusion from the rest of the sample.
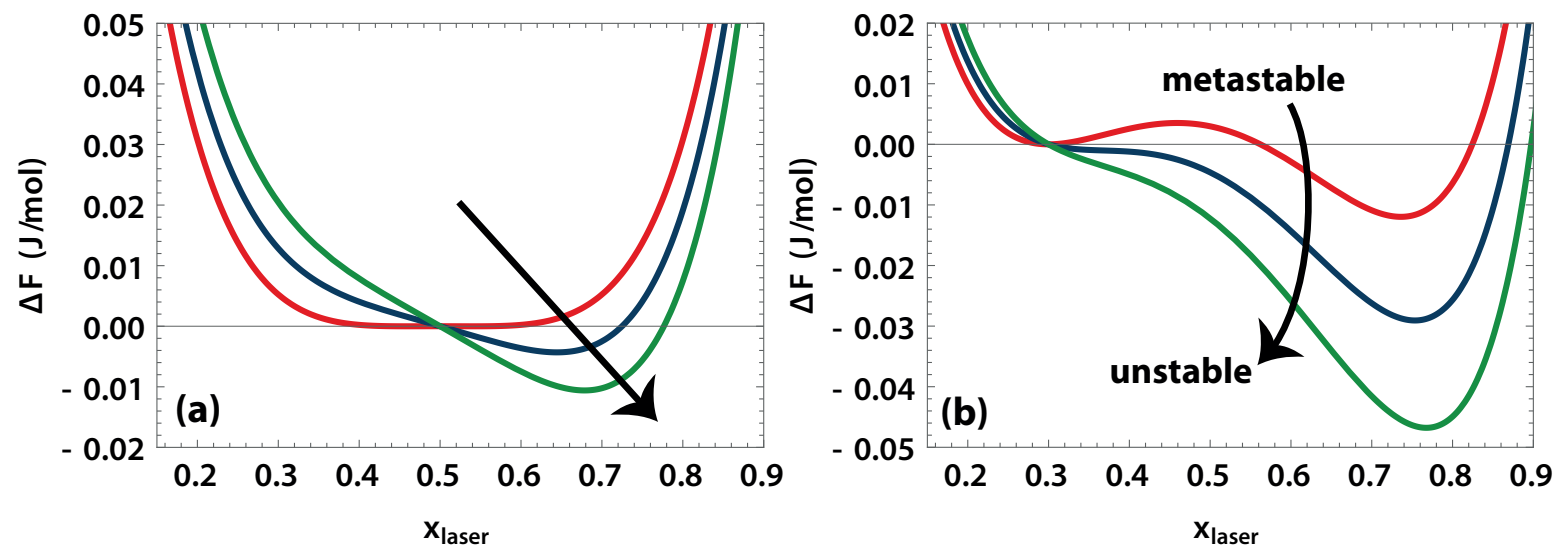

Figure 2. Summary of the laser-induced phase separation (LIPS) and laser-induced nucleation experiments. (a) A plot of the change in free energy $\Delta F$ in a liquid mixture of mole fraction $x_{0}$ when the mole fraction is changed to $x_{\text {laser }}$ in a small volume. Here the initial mole fraction is $x_{0}=0.5$, the laser intensity is increased from red to blue to green. When the laser power is increased, the minimum in the free-energy potential shifts to higher mole fraction causing phase separation. (b) As in (a) but with initial mole fraction $x_{0}=0.3$ and for a metastable mixture. When the laser is off, a free-energy barrier prevents the system from phase separating. At high laser power the barrier disappears, causing the system to transition from metastable to unstable.

Experiments were carried out on nitrobenzene-decane, aniline-cyclohexane, nitromethane-octanol, and phenol-decane mixtures. All these mixtures have upper consolute temperatures near room temperature. Samples were held in a cryogenically cooled stage mounted in a modified microscope, ${ }^{26,43,44}$ which allowed a $785-\mathrm{nm}(<200 \mathrm{~mW})$ or $1047-\mathrm{nm}$ $(<8 \mathrm{~W})$ laser beam to be focused in the sample.

When a laser is focused in the fully mixed sample while it is being observed by phase-contrast microscopy, a bright spot becomes visible (Figure 3). This shows that laser-induced phase separation (LIPS) takes place and that the fraction that is separated out of the mixture has a higher refractive index as confirmed by fluorescence microscopy experiments. ${ }^{41}$ Temperature dependent experiments show that LIPS strongly increases in strength on cooling as the binodal is approached and concentration dependent experiments show that the effect maximizes near the liquid-liquid critical point.

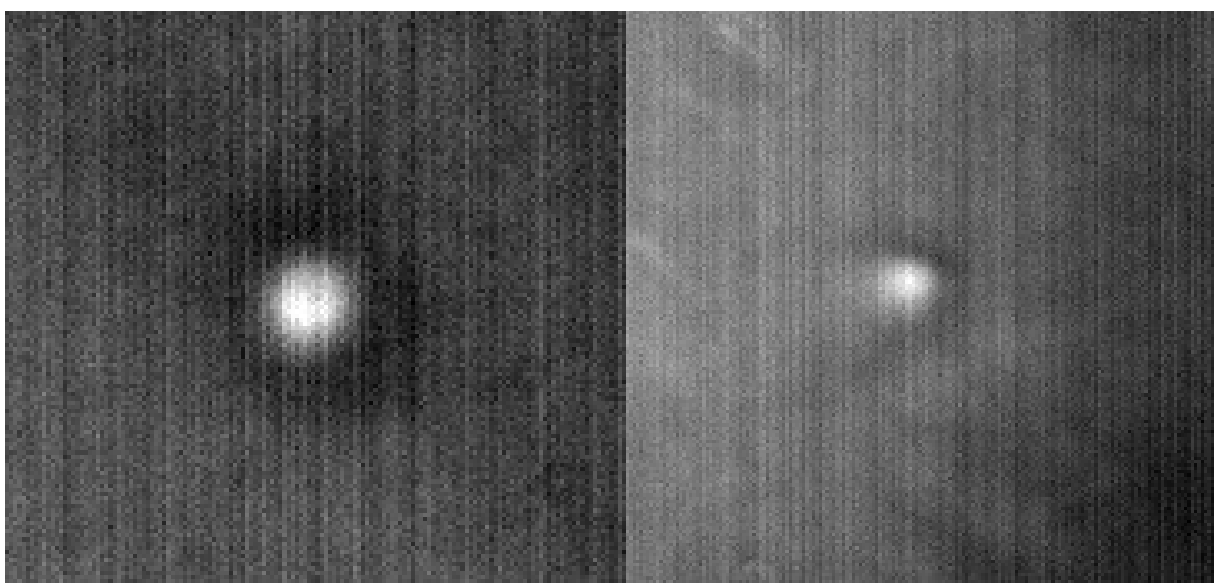

Figure 3. Laser-induced phase separation (LIPS) in aniline-cyclohexane (left) and phenol-decane (right). 
Nucleation induced by LIPS could be demonstrated when the temperature was set on the binodal temperature for a given mole-fraction $x$. When the laser is switched on, a LIPS droplet is seen in phase-contrast imaging (see Figure 4). Switching the laser off triggers the nucleation of phase-separated droplets that ripen into a single droplet that slowly (minutes) disappears again through remixing. This effect can be understood as the interplay of LIPS, diffusion, and heating. ${ }^{41}$

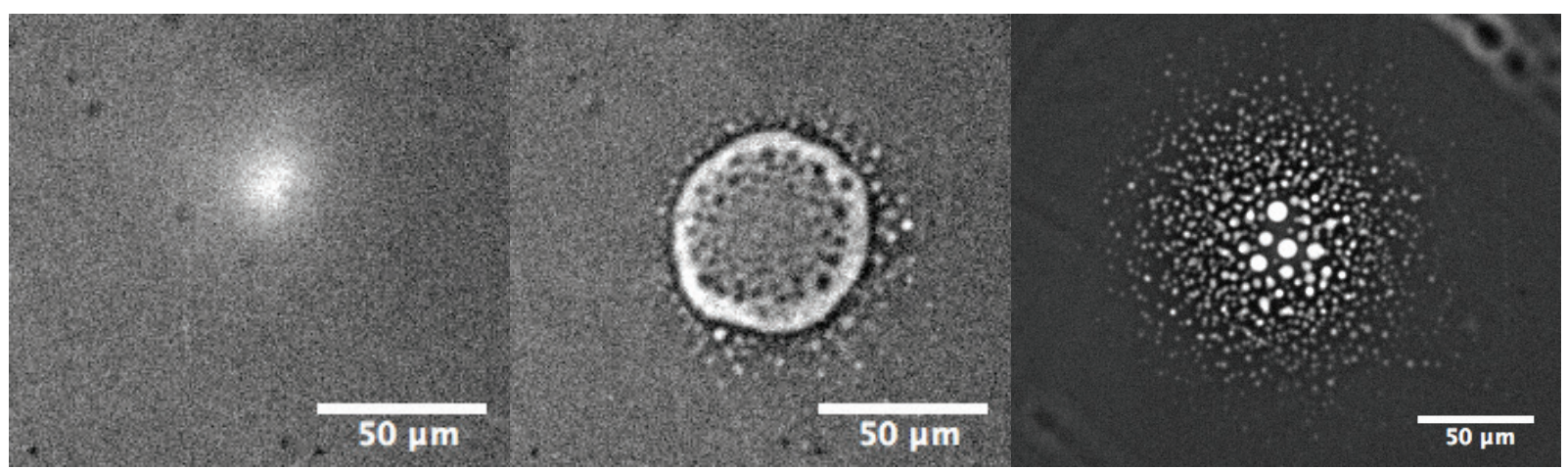

Figure 4. Laser-induced nucleation triggered via the LIPS effect in nitrobenzene-decane. (left) In the mixed regime near a binodal, LIPS produces a droplet enriched in nitrobenzene. (middle) Switching off the laser triggers nucleation, followed by Ostwald ripening. (right) As before but for longer LIPS period (120 s vs. $60 \mathrm{~s})$.

\section{CONCLUSIONS}

Near phase-transition boundaries (liquid-gas, liquid-solid, liquid-liquid, etc.), concentration fluctuations will occur that maximize near a critical point. We have shown that an optical-tweezing potential, only relying on the stored electromagnetic energy in the laser focus, can control such fluctuations to enrich one component in a mixture or to trigger the nucleation of a phase-separated droplet by placing the mixture below the binodal.

This effect is different from laser-induced photothermal phase separation, which relies on liquid mixtures with a lower consolute boundary that is crossed through a trivial heating effect. ${ }^{45-47}$ The LIPS effect is similar to but much larger than the optically biased diffusion of molecules described previously, which is only significant for molecules excited on resonance. ${ }^{48}$ Since nearly all crystals have a higher refractive index than their corresponding solution, this effect can in principle explain all previous laser-induced crystal-nucleation results.

The effect described here does not depend on the presence of pre-nucleation clusters ${ }^{36}$ that can be trapped or tweezed by the laser. ${ }^{49}$ Instead, it creates an optical-tweezing potential that lowers the free energy of the phase-separated state enhancing the probability for nucleation. This is a generic effect but will be enhanced near a liquid-liquid critical point. Phase manipulation and nucleation can be induced with a straightforward low-power laser diode suggesting future practical applications in the manipulation of matter.

\section{ACKNOWLEDGMENTS}

We thank the Engineering and Physical Sciences Research Council (EPSRC) for support through grants EP/J004790/1, $\mathrm{EP} / \mathrm{J} 009733 / 1$, and EP/N007417/1.

\section{REFERENCES}

[1] Garetz, B., Aber, J., Goddard, N. et al., "Nonphotochemical, polarization-dependent, laser-induced nucleation in supersaturated aqueous urea solutions," Phys. Rev. Lett. 77(16), 3475 (1996).

[2] Garetz, B., Matic, J., and Myerson, A., "Polarization switching of crystal structure in the nonphotochemical lightinduced nucleation of supersaturated aqueous glycine solutions," Phys. Rev. Lett. 89(17), 175501 (2002).

[3] Sun, X., Garetz, B., and Myerson, A., "Supersaturation and polarization dependence off polymorph control in the nonphotochemical laser-induced nucleation (nplin) of aqueous glycine solutions," Cryst. Growth. Des. 6(3), 684 (2006). 
[4] Ward, M. R., Mchugh, S., and Alexander, A. J., "Non-photochemical laser-induced nucleation of supercooled glacial acetic acid," Phys. Chem. Chem. Phys. 14(1), 90 (2012).

[5] Iefuji, N., Murai, R., Maruyama, M. et al., "Laser-induced nucleation in protein crystallization: Local increase in protein concentration induced by femtosecond laser irradiation," J. Cryst. Growth 318(1), 741 (2011).

[6] Liu, Y., Van Den Berg, M. H., and Alexander, A. J., "Supersaturation dependence of glycine polymorphism using laser-induced nucleation, sonocrystallization and nucleation by mechanical shock," Phys. Chem. Chem. Phys. 19(29), 19386 (2017).

[7] Duffus, C., Camp, P. J., and Alexander, A. J., "Spatial control of crystal nucleation in agarose gel," J. Am. Chem. Soc. 131(33), 11676 (2009).

[8] Li, W., Ikni, A., Scouflaire, P. et al., "Non-photochemical laser-induced nucleation of sulfathiazole in a water/ethanol mixture," Cryst. Growth. Des. 16(5), 2514 (2016).

[9] Usman, A., Uwada, T., and Masuhara, H., "Optical reorientation and trapping of nematic liquid crystals leading to the formation of micrometer-sized domain," J. Phys. Chem. C 115(24), 11906 (2011).

[10] Kosa, T., Sukhomlinova, L., Su, L. et al., "Light-induced liquid crystallinity," Nature 485(7398), 347 (2012).

[11]Knott, B. C., Larue, J. L., Wodtke, A. M. et al., "Communication: Bubbles, crystals, and laser-induced nucleation,” J. Chem. Phys. 134(17), 171102 (2011).

[12] Knott, B. C., Duff, N., Doherty, M. F. et al., "Estimating diffusivity along a reaction coordinate in the high friction limit: Insights on pulse times in laser-induced nucleation," J. Chem. Phys. 131(22), 224112 (2009).

[13] Knott, B. C., Doherty, M. F., and Peters, B., "A simulation test of the optical kerr mechanism for laser-induced nucleation,” J. Chem. Phys. 134(15), 154501 (2011).

[14]Liu, Y., Ward, M. R., and Alexander, A. J., "Polarization independence of laser-induced nucleation in supersaturated aqueous urea solutions," Phys. Chem. Chem. Phys. 19(5), 3464 (2017).

[15] Yuyama, K.-I., Rungsimanon, T., Sugiyama, T. et al., "Selective fabrication of $\alpha$ - and $\gamma$-polymorphs of glycine by intense polarized continuous wave laser beams," Cryst. Growth. Des. 12(5), 2427 (2012).

[16] Sugiyama, T., Yuyama, K.-I., and Masuhara, H., "Laser trapping chemistry: From polymer assembly to amino acid crystallization," Acc. Chem. Res. 45(11), 1946 (2012).

[17] Yuyama, K.-I., Sugiyama, T., and Masuhara, H., "Laser trapping and crystallization dynamics of 1-phenylalanine at solution surface," J. Phys. Chem. Lett. 4(15), 2436 (2013).

[18] Yuyama, K.-I., Ueda, M., Nagao, S. et al., "A single spherical assembly of protein amyloid fibrils formed by laser trapping," Angew. Chem. Int. Ed. 129(24), 6843 (2017).

[19] Niinomi, H., Sugiyama, T., Miyamoto, K. et al., ““Freezing” of naclo 3metastable crystalline state by optical trapping in unsaturated microdroplet," Cryst. Growth. Des. 18(2), 734 (2018).

[20]Bowman, R. W., and Padgett, M. J., "Optical trapping and binding," Rep. Prog. Phys. 76(2), 026401 (2013).

[21] Bartkiewicz, S., and Miniewicz, A., "Whirl-enhanced continuous wave laser trapping of particles," Phys. Chem. Chem. Phys. 17(2), 1077 (2014).

[22] Gebauer, D., and Coelfen, H., "Prenucleation clusters and non-classical nucleation," Nano Today 6(6), 564 (2011).

[23] Davey, R. J., Schroeder, S. L. M., and Ter Horst, J. H., "Nucleation of organic crystals--a molecular perspective.," Angew. Chem. Int. Ed. 52(8), 2166 (2013).

[24] Gebauer, D., Voelkel, A., and Coelfen, H., "Stable prenucleation calcium carbonate clusters," Science 322(5909), 1819 (2008).

[25] Reichenbach, J., and Wynne, K., "Frustration vs. Prenucleation: Understanding the surprising stability of supersaturated sodium thiosulfate solutions," J. Phys. Chem. B in press, (2018).

[26] Mosses, J., Turton, D. A., Lue, L. et al., "Crystal templating through liquid-liquid phase separation," Chem. Commun. 51, 1139 (2015).

[27] Pouget, E. M., Bomans, P. H. H., Goos, J. a. C. M. et al., "The initial stages of template-controlled caco3 formation revealed by cryo-tem.," Science 323(5920), 1455 (2009).

[28] Dey, A., Bomans, P. H. H., Mueller, F. A. et al., "The role of prenucleation clusters in surface-induced calcium phosphate crystallization," Nat. Mater. 9(12), 1010 (2010).

[29] Smeets, P. J. M., Finney, A. R., Habraken, W. J. E. M. et al., “A classical view on nonclassical nucleation.,” Proc. Natl. Acad. Sci. U.S.A. 114(38), E7882 (2017).

[30] Van Driessche, A. E. S., Van Gerven, N., Bomans, P. H. H. et al., "Molecular nucleation mechanisms and control strategies for crystal polymorph selection," Nature 556(7699), 89 (2018). 
[31] Tenwolde, P., and Frenkel, D., "Enhancement of protein crystal nucleation by critical density fluctuations," Science 277(5334), 1975 (1997).

[32] Wedekind, J., Xu, L., Buldyrev, S. V. et al., "Optimization of crystal nucleation close to a metastable fluid-fluid phase transition,” Sci. Rep. 5(1), 1 (2015).

[33] Xu, L., Buldyrev, S. V., Stanley, H. E. et al., "Homogeneous crystal nucleation near a metastable fluid-fluid phase transition," Phys. Rev. Lett. 109(9), 95702 (2012).

[34]Bonnett, P., Carpenter, K., Dawson, S. et al., "Solution crystallisation via a submerged liquid-liquid phase boundary: Oiling out," Chem. Commun.(6), 698 (2003).

[35] Takasuga, M., and Ooshima, H., "Control of crystal size during oiling out crystallization of an api," Cryst. Growth. Des. 14(11), 6006 (2014).

[36] Gebauer, D., Kellermeier, M., Gale, J. D. et al., "Pre-nucleation clusters as solute precursors in crystallisation.," Chem. Soc. Rev. 43(7), 2348 (2014).

[37] Wallace, A. F., Hedges, L. O., Fernandez-Martinez, A. et al., "Microscopic evidence for liquid-liquid separation in supersaturated caco3 solutions.," Science 341(6148), 885 (2013).

[38] Casner, A., and Delville, J.-P., "Giant deformations of a liquid-liquid interface induced by the optical radiation pressure,” Phys. Rev. Lett. 87(5), 4853 (2001).

[39] Schroll, R. D., Wunenburger, R., Casner, A. et al., "Liquid transport due to light scattering," Phys. Rev. Lett. 98(13), 2355 (2007).

[40] Katsir, Y., and Tsori, Y., "Recent advances in liquid mixtures in electric fields.," Journal of physics. Condensed matter : an Institute of Physics journal 29(6), 063002 (2017).

[41] Walton, F., and Wynne, K., "Control over phase separation and nucleation using a laser-tweezing potential," Nat. Chem. 10, 506 (2018).

[42] Jones, R. a. L., [Soft condensed matter] Oxford University Press, Oxford(2002).

[43] Mosses, J., Syme, C. D., and Wynne, K., "Order parameter of the liquid-liquid transition in a molecular liquid," J. Phys. Chem. Lett. 6, 38 (2015).

[44] Syme, C. D., Mosses, J., González Jiménez, M. et al., "Frustration of crystallisation by a liquid-crystal phase," Sci. Rep. 7, 42439 (2017).

[45]Hofkens, J., Hotta, J., Sasaki, K. et al., "Molecular assembling by the radiation pressure of a focused laser beam: Poly( n-isopropylacrylamide) in aqueous solution," Langmuir 13(3), 414 (1997).

[46] Oana, H., Kishimura, A., Yonehara, K. et al., "Spontaneous formation of giant unilamellar vesicles from microdroplets of a polyion complex by thermally induced phase separation," Angew. Chem. Int. Ed. 48(25), 4613 (2009).

[47] Kitamura, N., Yamada, M., Ishizaka, S. et al., "Laser-induced liquid-to-droplet extraction of chlorophenol: Photothermal phase separation of aqueous triethylamine solutions," Anal. Chem. 77(18), 6055 (2005). [48] Osborne, M. A., Balasubramanian, S., Furey, W. S. et al., "Optically biased diffusion of single molecules studied by confocal fluorescence microscopy," J. Phys. Chem. B 102(17), 3160 (1998).

[49] Masuhara, H., Sugiyama, T., Yuyama, K.-I. et al., "Optical trapping assembling of clusters and nanoparticles in solution by cw and femtosecond lasers," Opt. Rev. 22(1), 143 (2015). 